\title{
A Functional-Structural Model of Rice Seedling Coupled with Nitrogen Metabolism
}

\author{
Zhuoyang Yusang ${ }^{1}$, Lifeng $\mathrm{Xu}^{1}$, Weilong Ding ${ }^{1}$, Zhangyun $\mathrm{Yi}^{2}$ and Yunwei Qiu ${ }^{1}$ \\ 1. College of Computer Science \& Technology, Zhejiang University of Technology, Hangzhou 310023, P. R. China \\ 2. School of Biosystems Engineering and Food Science, Zhejiang University, Hangzhou 310058, P. R. China
}

Received: July 28, 2014 / Published: August 20, 2014.

\begin{abstract}
With the ability of representing the association and inner-feedback between plant morphological structure and physiological functions, functional-structural plant modeling (FSPM) approach has been used in many works, trying to better understand the mechanisms of integrating plant functions and its structure, and their communication with environmental factors. To do so, an FSPM of rice seedling was developed in this study, including structural morphogenetic model, photosynthetic model and biomass partitioning module. It can thus describe the developmental course of the rice structure dynamically based on the processes of biomass producing and partitioning. Furthermore, the processes of nitrogen metabolism, which influence the $\mathrm{N}$ content and growth dynamics of the virtual rice, were also considered. The model was developed with L-system on a platform established with Java programming language, which took over the parsing and visualization of the L-system strings to 3D objects using Java 3D extended library. The physiological processes in the model can be modified and further improved to gradually meet the needs for modeling the whole life cycle of rice, e.g., considering the respiration, and interaction with other environmental factors like $\mathrm{CO}_{2}$, temperature, etc.. The model was developed to provide a platform to systematically study and understand how plant systems like rice seedling work. The model and the virtualization platform can be expanded to provide decision support on $\mathrm{N}$ fertilizer application for the rice seedling and the other crops.
\end{abstract}

Key words: Functional-structural plant model, rice seedling, nitrogen metabolism, L-system, visualization, plant model.

\section{Introduction}

In recent years, functional-structural plant modelling (FSPM) has become one of the most significant branches of virtual plant. Differing from the conventional plant modeling methods, which focused either on process-based functions or plant structure, FSPM constructs a model with plant physiology and morphology both integrated [1]. It is able to simulate the dynamics of plant growth based on the processes of assimilate production and partitioning. It is thus used in a variety of areas such as education, studies of virtual breeding and yield prediction [2].

Corresponding author: Lifeng Xu, Ph.D., research fields: functional-structural plant modeling, virtual plant and bioinformatics. E-mail: lfxu@zjut.edu.cn.
Concerning plant morphological structure, L-system [3] proposed by Lindenmayer in 1968 is currently the most widely used. An L-system is a parallel rewriting system, which consists of an alphabet of symbols, an initial Axiom string, and a set of rules which define the way that symbols can be replaced recursively. A series of improvements based on L-system such as Open L-system [4] and differential L-system [5] are proposed. With the capability of modeling the morphology of a variety of organisms and describing the growth behavior, L-systems have great advantages of modeling the growth process of plant.

As a part of virtual plant study, a lot of modeling platforms have been developed, most of which are based on L-system. Typical cases are L-studio [6] 
developed by scientists in the University of Calgary, and GroIMP [7], in which an eXtended L-system language (XL) is used. L-studio includes two embedded L-system based simulators, i.e., one as a fractal generator with continuous parameters with the foundation of L-system, and the other as a modeling program that the models are developed using $\mathrm{L}+\mathrm{C}$ modeling language which adds L-system specific constructors to $\mathrm{C}++$ programming language. GroIMP is also compatible with Java language. Based on this platform, Chen et al. [8] developed a visual cotton model for above-ground organs. Xu et al. [9] proposed a functional-structural model of rice, with a genetic module integrated. Thus the dynamics of development of plant height was partially controlled by the quantitative genetic factors they introduced.

However, it still remains a challenge to fully understand the mechanisms of plant growth dynamics considering environmental factors with functional-structural plant models. The objective of the paper is to present a case study of a functional-structural model of rice seedling focusing on two aspects: combination of the plant architecture (structure) and functions (physiology), and influences of plant growth caused by various nitrogen concentration of the culture media. In this study, the 3-demensional structure of the rice seedling was reconstructed based on the physiological processes. The physiological aspect of the model contains a photosynthesis module and an assimilated partitioning module. And the processes of the nitrogen metabolism were also integrated into the model to compute the dynamics of nitrogen content for the rice seedling. Consequently, the dynamics of nitrogen content of the environment led to the changes of the growth processes, accumulating the various representation of the seedling appearance on plant level. Furthermore, it can be expanded to provide decision support on nitrogen fertilizer application for the rice seedling and the other crops by analyzing the physiological status at different time.

\section{Materials and Methods}

\subsection{Scope of the Model}

The model was written with the modeling language of L-system using a platform that implemented with Java language and Java 3D library. The plant structure was thus described with L-system strings. The rules of plant growth in the model were applied to determine how the plant topology and organs change during the period of growth. And the physiological aspects were encapsulated with functions.

The organs of a rice seedling contain leaf blades and sheaths, both formed from the buds. The underground part (root system) was neglected in the current model.

\subsection{Platform Implementation}

\subsubsection{Overall Design}

The modeling platform was implemented with Java language and Java 3D extended library. There are three major modules: GUI (graphical user interface) module, data processing module, and $3 \mathrm{D}$ visualization module. The latter two took charge of parsing L-system code to visual 3D graphic objects.

The data processing module consists of a parser and a repository, including an environmental model and an inference machine. Like the most of the modeling platforms [6, 7], the growth rules from the code are parsed, and then functions of physiology are executed. Environmental model is able to simulate the processes of interaction between environmental factors and plant model. Specific physiological parameters are then transferred to the inference machine for analyzing the physical health of plants.

The 3D visualization module consists of two units: organ construction unit responsible for forming plant organs, and L-system visualization unit for converting the L-system strings describing plant topology into a 3-dementional model.

The workflow of execution steps for the rules is shown in Fig. 1. The growth period of the virtual rice 


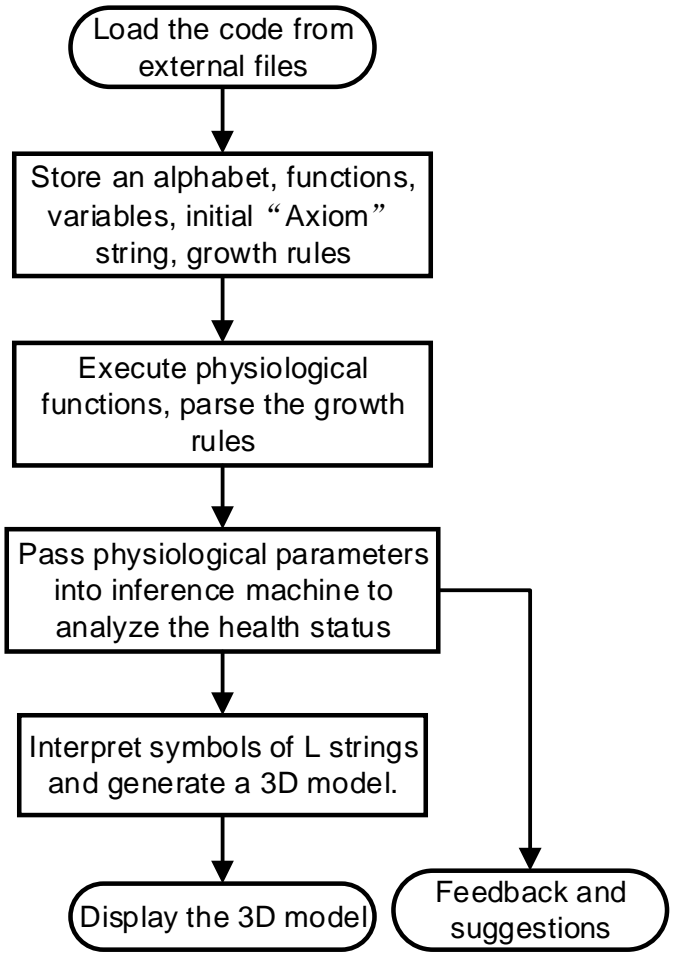

Fig. 1 Diagram representing the work flow of the modelling platform.

seedling was described in time steps. For each time step, the L-system-based rules (query, replacement, etc.) were applied to dynamically generate the 3-demensional structure of the plant.

\subsubsection{Visualization of L-system Strings}

Topological structure of rice seedling is described with L-system strings consisting of a set of symbols within an alphabet. Hence, plant architecture can be modeled via interpreting symbols of L-system strings. An L-system string consists of four types of symbols:

Graphical object: each symbol of this type represents a graphical object with two attributes: 3D coordinates in space, and the direction with respect to the XYZ axis, e.g., "F $(1,0.05)$ " represents a cylinder with height of one and radius of 0.05 . These two attributes which determine the location of the graphical object in 3-dementional space are stored in a matrix.

Stack manipulation: including "[" and "]". "[" is used to push the graphical node into the stack, whereas "]" is used to pop the graphical node out of the stack and make it to be the current node.

Rotation: each symbol of this type means rotating a certain degrees around one of the $\mathrm{XYZ}$ axis, e.g., " $\mathrm{RH}$ (60)" means rotating 60 degrees around $\mathrm{Y}$ axis.

Other symbols: each symbol of this type is only used as a label for replacement with the growth rules and has no specific meanings.

The overall algorithm is shown as following:

Initialize an empty graphical node as the root node to store the plant model, a matrix to store the spatial information including the location and direction, and a stack to store the graphical nodes.

Traverse the L-system string from left to right, when the graphical objects are found, skip to step 3 . When the symbols for stack manipulation are found, skip to step 4. When the rotation symbols are found, skip to step 5. Do nothing when the other symbols are found. If the traversal is completed, skip to step 6 .

Generate a new graphical node with the corresponding graphical object in it and set it to be the current node.

When "[" is found, push the current node into the stack. When "]" is found, pop the node at the top of the stack out and set it to be the current node.

Read the rotation degree from the string and call the corresponding methods to rotate.

Return the root node which contains the plant model.

\subsection{Construction of Functional-Structural Model of Rice Seedling}

A FSPM of rice seedling was developed with the modeling platform. The rice model consists of two sub-models: morphological model and physiological model. The morphological model was established through constructing the 3D model of seedling organs and generating the topological structure described with the L-system strings. The physiological model was based on the concept of source-sink relationship [10]. A photosynthesis module and an assimilated partitioning module were integrated via a concept 
called "assimilate pool", where assimilate was accumulated, and used to calculate the dynamics of assimilate produced and allocated into organs, respectively. In this way, the dynamics of assimilate allocated into organs lead to the changes of the organ size. Besides, there was also a nitrogen metabolism module combined within the physiological model, influencing the photosynthesis process of the virtual plant (described in Section 2.3.4). The relationships between the above mentioned modules are shown in Fig. 2.

\subsubsection{Modeling of Organs}

The geometrical model of leaf sheath was simulated using cylinder. And Bezier surface was used to simulate leaf blade, as shown in Fig. 3.

The procedure of modeling the geometrical shape of leaf blade can be separated into 3 steps:

Create a $17 \times 17$ grid which forms the surface of blade and a $4 \times 4$ grid to control all vertices of the surface.

In each row of the $4 \times 4$ grid, input four vertices in that row and calculate all 17 vertices of that row in the $17 \times 17$ grid using deCasteljau algorithm [11]

In each column of the $17 \times 17$ grid, input four vertices of that column and calculate all 17 vertices of that column using deCasteljau algorithm to get all vertices of the surface.

A leaf photo taken with Nikon ${ }^{\circledR}$ D7000 was used as textures and applied to the leaf model. In order to get distinct appearance of individuals with different level of $\mathrm{N}$ content (defined as different health conditions in the model), these textures were modified with Adobe ${ }^{\circledR}$ Photoshop 10.0 to get various colors.

\subsubsection{Generation of the Topological Structure}

There were two types of organs considered in the rice seedling model, i.e., leaf blade and sheath. For further optimizing and simplifying the processes of modelling, the modelling language was tailored based on L-system specific for the platform.

Different graphical objects were defined as:

(1) module M (length, width, age, order) extends leaf model.

(2) module F (length, radius, age, order) extends cylinder.

Where, $\mathrm{M}$ and $\mathrm{F}$ were objects representing two organs: leaf blade and leaf sheath.

And a symbol with an age parameter was used to represent the buds and also set to be the initial axiom string. Ages of organs and ages of the buds were used to specify the start time and stop time of growth.

Two rules of growth were defined and applied to the buds and leaf organs:

(1) If the bud grows to the age when a new branch should appear, it will be replaced by a new leaf.

(2) The size of an organ increases with the time until it reaches a certain age.

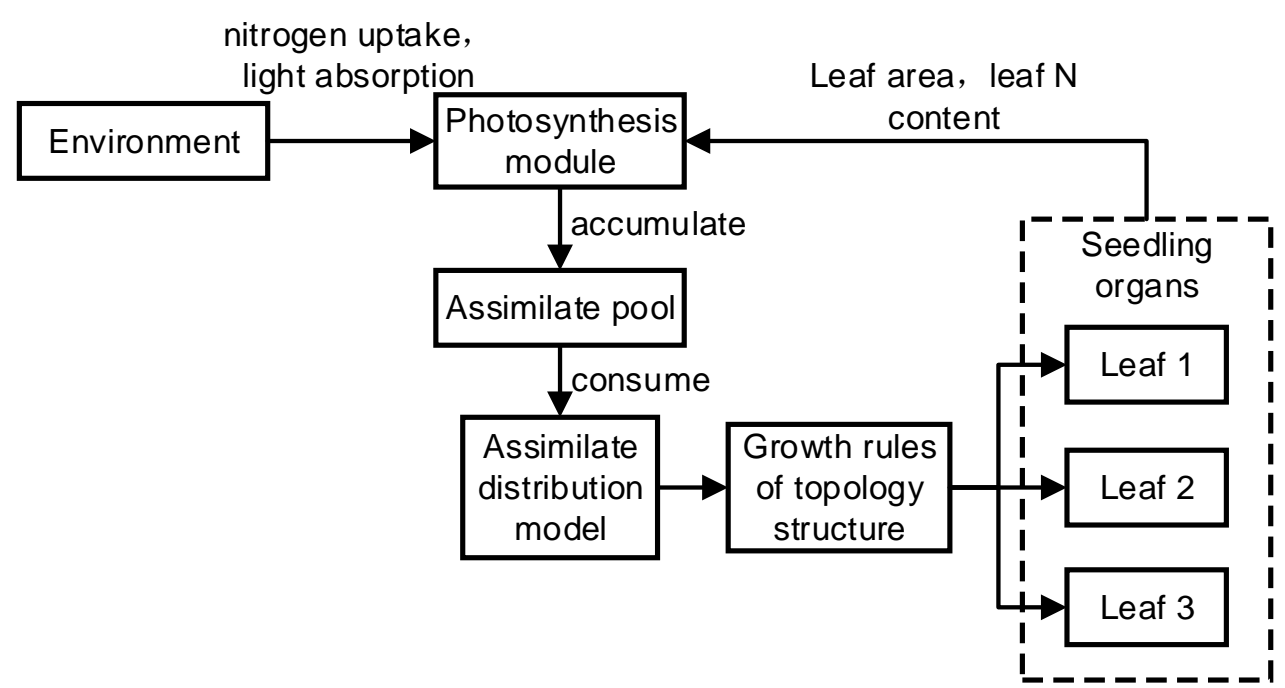

Fig. 2 Relationship and association between different modules within the rice seedling model. 


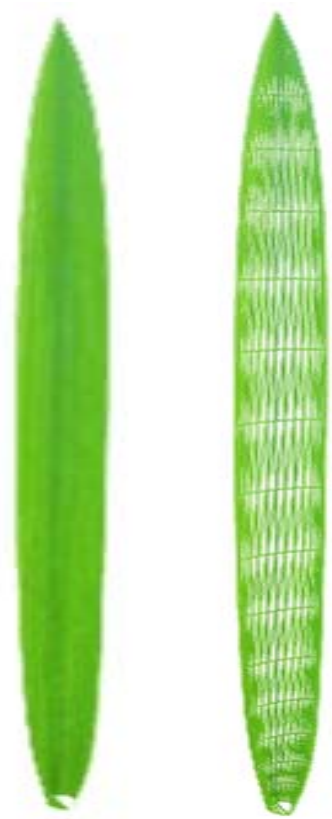

Fig. 3 Simulation results of the leaves: a textured image of a leaf blade (left), and a blade in wireframe mode (right).

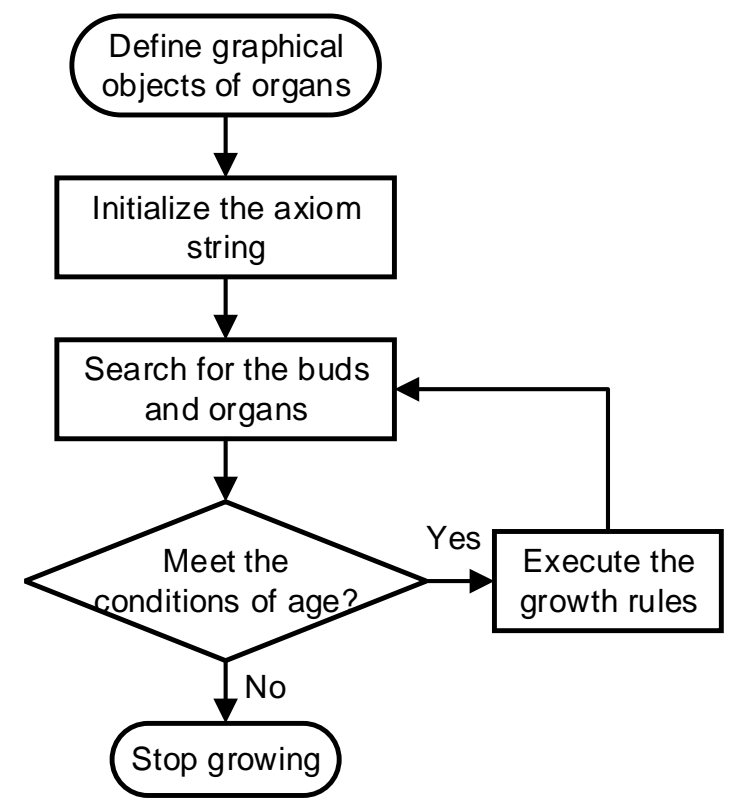

Fig. 4 Steps of simulation of dynamic morphological processes of rice seedling in the model.

The steps of growth are shown in Fig. 4. Rules were iteratively applied to the initial axiom string. Each time step during the iteration represents one day in the growth period of the seedling, i.e., one step in simulation means one day in plant growth.

2.3.3 Modeling of the Physiological Processes

(1) Photosynthesis model
In this model, assimilate produced by photosynthesis is accumulated in an assimilate pool (ap), and allocated into organs during the process of assimilate partitioning. For the computation of net photosynthesis rate, the model proposed by Keisuke Ono [12] was used to model the leaf photosynthesis. A fixed value $\left(500 \mu \mathrm{mol} / \mathrm{m}^{2} \cdot \mathrm{s}\right.$ [12] was used as the light absorption, and nitrogen content of leaf was set to be the factor influencing the photosynthesis rate.

With this model, the accumulative process of assimilate pool was simulated using the following equation:

$$
a p_{t}=a p_{t-1}+A_{g} \cdot G L A \cdot \Delta t
$$

where $a p_{t}$ is the assimilate accumulated on day $t, a p_{t-1}$ is the assimilate accumulated on day $t-1, A_{g}$ represents the net photosynthesis rate, GLA is green leaf area, $\triangle t$ is the length of a growth time step (one day).

(2) Assimilate partitioning model

The process of assimilate partitioning is actually the process that organs compete for assimilate produced by photosynthesis [10]. In the seedling model, the products are allocated into growing leaves.

Based on the concept of source-sink relationship mentioned above, the distribution ratio of assimilate production $\left(f_{i}\right)$ can be calculated as:

$$
f_{i}=\frac{S_{i}}{\sum S_{i}}
$$

where, $S_{i}$ represents the sink strength of an organ, $\sum S_{i}$ is total sink strength. Beta function [13] which is able to calculate the potential growth rate was invoked and carried out to simulate the sink strength of each organ. By entering four parameters: the time when growth ends $\left(t_{e}\right)$, the time when the growth rate reaches a maximum $\left(t_{m}\right)$, the final size of the organ $\left(w_{\max }\right)$, and the current time $t$, the potential growth rate of each organ can be calculated. The leaf parameters were obtained by destructive measurement, as shown in Table 1.

The data containing parameters of three leaves were used to calculate the potential growth rate (sink strength) of the leaves. 
Table 1 Parameters used in the growth function for leaves.

\begin{tabular}{lllll}
\hline \multirow{2}{*}{ Organs } & $t_{e}(\mathrm{~d})$ & \multirow{2}{*}{$t_{m}(\mathrm{~d})$} & \multicolumn{2}{c}{$w_{\max }(\mathrm{cm})$} \\
\cline { 4 - 5 } & & & length & width \\
\hline Leaf 1 & 4 & 8 & 1.20 & 0.26 \\
Leaf 2 & 9 & 16 & 6.40 & 0.30 \\
Leaf 3 & 15 & 23 & 8.90 & 0.34 \\
\hline
\end{tabular}

For the computation of obtained assimilate of each organ, the following equation was used:

$$
a s S_{i}=f_{i} \cdot a p
$$

where, $\operatorname{ass}_{i}$ represents the assimilate allocated into organ $i$.

And an updated assimilated pool at time $\mathrm{t}\left(a p_{t}\right)$ can be expressed as:

$$
a p_{t}=a p_{t-1}-a s s_{i} \cdot \Delta t
$$

By using a fixed coefficient $\left(2.3 \mathrm{mg} / \mathrm{cm}^{2}\right)$ converting the obtained assimilate production into the variation of leaf size, the dynamics of leaf length can be simulated.

\subsubsection{Modeling of Nitrogen Metabolism}

Nitrogen content of the environment is a critical factor influencing the assimilate production [14]. In the model, processes of nitrogen metabolism consisting of nitrogen absorption and consumption were considered. A method, Mchaelis-Menten equation [15], specially designed for conditions of hydroponics or sand culture, was used to compute the rate of nitrogen absorption:

$$
I_{N}=\frac{V_{\max } \cdot C}{K_{m}+C}
$$

$I_{N}$ is the actual absorption rate of nitrogen, $V_{\max }$ is the maximum absorption rate of nitrogen, $C$ is the nitrogen concentration of culture media, and $K_{m}$ is the affinity for nitrogen. For the computation of nitrogen consumption, a coefficient of utilization rate of nitrogen is used and set to be $10.0 \mathrm{~g}$ assimilate per $\mathrm{g}$ nitrogen [14]. The process of nitrogen consumption is thus calculated using the following equation:

$$
N_{C}=\frac{a S S}{10.0}
$$

where, ass represents the assimilate production, and
$N_{c}$ is consumed nitrogen.

The processes of nitrogen absorption and consumption can thus be computed with the equations discussed above, leading to the variation of nitrogen content of rice seedling in different time and environments. Hence, the net photosynthesis rate fluctuated according to the dynamics of nitrogen content of rice seedling. And the $\mathrm{N}$ content was chosen to be the factor for analyzing the health conditions of rice seedling in different time, which was further represented as leaves with different shape and color.

\section{Results and Discussion}

The result of the simulation of photosynthesis is shown in Fig. 5a. It is obvious that the assimilate production was rising during the growth period of the virtual rice seedling. The increasing rate of the production was accelerated during day 9 to day 18 , mainly caused by the sharp increase of leaf area. Fig. $5 \mathrm{~b}$ illustrates the dynamics of assimilate pool after biomass partitioning. From the day 5 to day 20, the stored amount of endosperm and assimilate production dropped sharply. It was due to the consumption of the assimilate production in the pool to form the first three fast growing leaves. And the rapid increase of leaf area led to the rise of the production rate of photosynthesis. An upward trend was thus presented in the last few days. Fig. 5c illustrates the length dynamics of the 3rd leaf. In the first 14 days, the growth rate was increasing, and then it tended to slow down. And Fig. 6 shows the dynamics of the seedling morphology considering processes of photosynthesis and assimilate partitioning.

The simulated assimilate produced by photosynthesis was minimum in the first 5 days and finally reached at $0.015 \mathrm{~g}$ on the last day. The assimilate pool began to decrease from $0.02 \mathrm{~g}$ on day 5 and fell to the bottom at $0.008 \mathrm{~g}$ on day 20 . In the end, it went up slightly to $0.012 \mathrm{~g}$. The growth rate of 


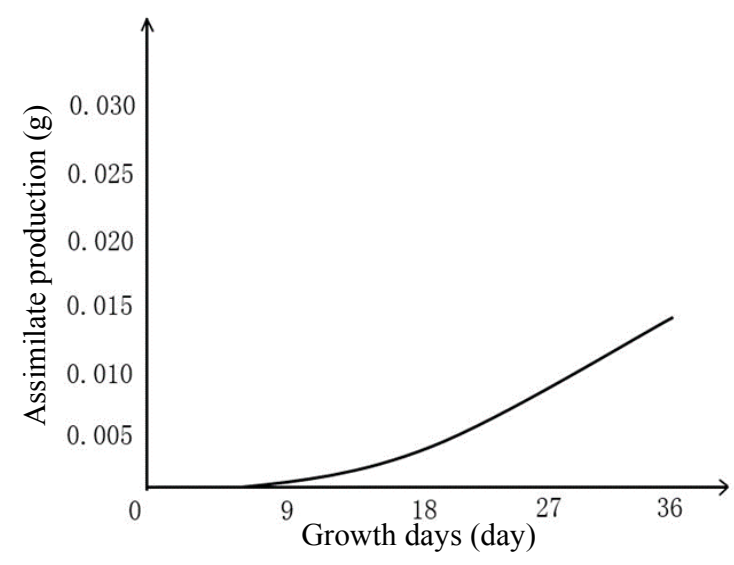

(a)

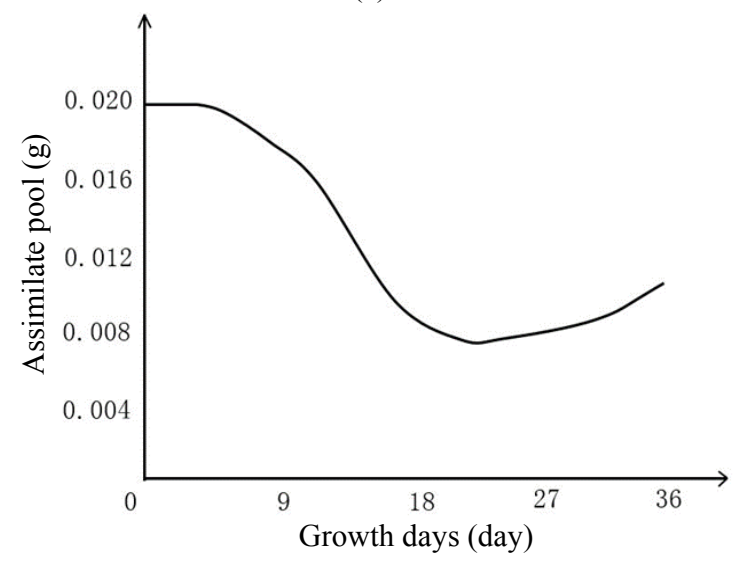

(b)

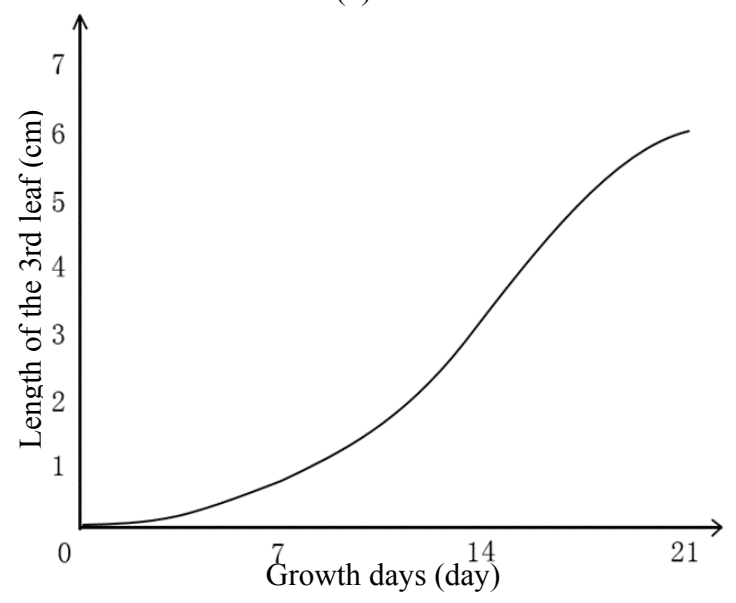

(c)

Fig. 5 Dynamics charts for (a) the accumulative biomass production, (b) size of assimilate pool (considering assimilate partitioning), and (c) the length of the third leaf.

the 3rd leaf reached a maximum on day 14 (counting from the start of its growth). And at last the length increased to $5.9 \mathrm{~cm}$.

Furthermore, the interaction between virtual plant

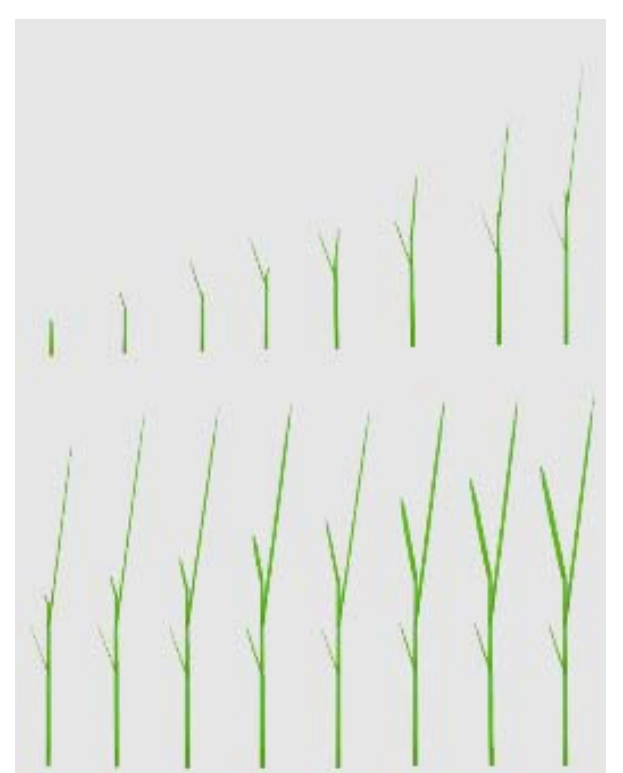

Fig. 6 Snapshots of the growth dynamics of a rice seedling individual from the modeling platform.

The first snapshot took from the 5th day of the growth as the first, to 35th as the last, and took one every two days between them.

and environmental inference ( $\mathrm{N}$ content) was also considered. Based on the integration of photosynthesis and assimilate partitioning, the processes of nitrogen metabolism were also simulated in the model. Thus the variations of nitrogen concentration of the environment can lead to the changes of photosynthetic rate and assimilate allocated into organs. A function describing the normal dynamics of nitrogen content was used for comparing with the current nitrogen content to evaluate the health condition. If the current $\mathrm{N}$ content is lower than the normal standard, a decision feedback will be given. If the rice seedling does not obtain enough nitrogen for supplement for a certain period of time, the seedling model will appear differently (e.g., leaf will bend and gradually turn yellow, as shown in Fig. 7).

However, connecting plant physiology and environmental factors to accurately simulate and predict the plant growth is still a tough topic. It is because both of internal mechanisms of plant and external environment are sophisticated. And competition between individuals in the population increases the difficulty of simulation. It is focused on 


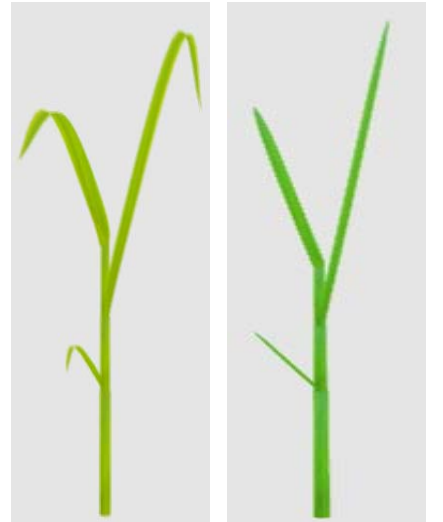

Fig. 7 A rice seedling individual of nitrogen deficiency (left), and an individual in normal status (right).

the above-ground parts of rice seedling in this study. While quantitative light absorption was fixed, and influences of other environmental factors such as moisture and temperature were ignored.

The rules for plant growth can be described with the modeling platform, thus to simplify the process of simulating morphology of the target plant. Moreover, the plant morphological structure model based on L-system is applicable to a variety of different physiological models, which makes it extensible.

\section{Conclusions}

A case study of developing a Functional-Structural model of rice seedling is presented. First, a modeling platform was established with Java programming language and Java 3D library. By interpreting the L-system symbols, a dynamic 3-demensional architecture of rice seedling was developed. The model integrated the morphological structure based on L-system and the physiological function model to simulate the growth process of rice seedling. Physiological model of rice seedling consists of photosynthesis module and assimilate partitioning module. And the nitrogen concentration of the growth environment was considered and implemented to influence the processes of nitrogen metabolism.

The present work is the initial step of the modeling work, ultimately aiming at linking physiological processes and selected environmental factors with a realistic 3D structural phenotype model of rice based on a platform that is simple but promising. In further studies, the structure model can be applied to simulate different growth periods of rice, with consideration of the interaction between plants and more environmental factors such as temperature, moisture and carbon dioxide concentration, improving the reliability and applicability of the model.

\section{Acknowledgments}

This work was partially funded by the National Natural Science Foundation of China under grant No. 31301230, and supported by scientific research fund of Zhejiang Provincial education department (No. Y201224867).

\section{References}

[1] Vos, J., Evers, J. B., Buck-Sorlin, G. H., Andrieu, B., Chelle, M., and de Visser, P. H. B. 2010. "Functional-Structural Plant Modelling: A New Versatile Tool in Crop Science." Journal of Experimental Botany 61 (8): 2101-15.

[2] Kang, M. Z. 2012. "Review and Perspectives on Research about Functional-structural Plant Models." Journal of System Simulation 24 (10): 2039-48. (in Chinese)

[3] Liu, G., and Zhou, G. M. 2008. "The Summarization of the Theory and Application of L-systems." Agriculture Network Information 9 (12): 21-3. (in Chinese)

[4] Prusinkiewicz, P., Hanan, J., and Měch, R. 1999. "An L-system-Based Plant Modeling Language." In Proceedings of AGTIVE 1999, Lecture Notes in Computer Science, 395-410.

[5] Prusinkiewicz, P., Hammel, M., and Mjolsness, E. 1993. "Animation of Plant Development." In Proceedings of SIGGRAPH 93, Computer Graphics, Annual Conference Series, 351-60.

[6] Prusinkiewicz, P., Karwowski, R., and Lane, B. 2007. "The L + C Plant-Modelling Language." In Functional-Structural Plant Modelling in Crop Production. Wageningen: Springer, 27-42.

[7] Kurth, W. 2007. "Specification of Morphological Models with L-systems and Relational Growth Grammars." Image-Journal of Interdisciplinary Image Science 5 (special issue): 1-25.

[8] Chen, C., Pan, X. B., Zhang, L. Z., and Pang, Y. M. 2012. "Functional and Structural Model for Above-Ground Growth in Cotton." Acta Agronomica Sinica 38 (12): 
2237-45. (in Chinese)

[9] Xu, J. J., Wang, B. H., Wu, Y. H., Du, P., Wang, J., Wang, M., Yi, C., Gu, M., and Liang, G. 2011. "Fine Mapping and Candidate Gene Analysis of Ptgms2-1, the Photoperiod-Thermo-Sensitive Genic Male Sterile Gene in Rice (Oryza sativa L.)." Theoretical and Applied Genetics 122 (2): 365-72.

[10] Liu, Y. H., Jia, H. K., and Gao, Q. 2006. "Review on Researches of Photoassimilates Partitioning and Its Models." Acta Ecologica Sinica 26 (6): 1981-6. (in Chinese)

[11] Zhang, H., and Daniel, L. Y. 2008. Computer Graphics Using Java $2 D$ and $3 D$. Beijing: Machine Press, 342. (in Chinese)

[12] Ono, K., Maruyama, A., Kuwagata, T., Mano, M., Takimoto, T., Hayashi, K., Hasegawa, T., and Miyata, A.
2013. "Canopy-Scale Relationships between Stomatal Conductance and Photosynthesis in Irrigated Rice." Global Change Biology 19 (7): 2212-3.

[13] Xu, L. F., Wei, Y., Ding, W. L., and Buck-Sorlin, G. 2013. "Study and Implementation of Functional-Structural Model of Rice GroIMP." Journal of System Simulation 25 (7): 1476-89. (in Chinese)

[14] Yang, J. P., Jiang, N., and Chen, J. 2002. "The Validation of Modeling Effects of Different Nitrogen Levels on the Leaf Nitrogen and Yield Dynamics of Rice." Plant Nutrition and Fertilizer Science 8 (3): 153-4. (in Chinese)

[15] Zhang, Y. L., Dong, Y. Y., Shen, Q. R., and Duan, Y. H. 2004. "Characteristics of $\mathrm{NH}_{4}{ }^{+}$and $\mathrm{NO}_{3}{ }^{-}$Uptake by Rices of Different Genotypes.” Acta Pedologica Sinice 41 (6): 919-23. (in Chinese) 